\title{
Robotic Autonomous Observatories: A Historical Perspective
}

\author{
Alberto Javier Castro-Tirado \\ Instituto de Astrofísica de Andalucía (IAA-CSIC), Glorieta de la Astronomía s/n, 18008 Granada, Spain \\ Correspondence should be addressed to Alberto Javier Castro-Tirado, ajct@iaa.es
}

Received 22 July 2009; Accepted 29 January 2010

Academic Editor: Lorraine Hanlon

Copyright (c) 2010 Alberto Javier Castro-Tirado. This is an open access article distributed under the Creative Commons Attribution License, which permits unrestricted use, distribution, and reproduction in any medium, provided the original work is properly cited.

This paper presents a historical introduction to the field of Robotic Astronomy, from the point of view of a scientist working in this field for more than a decade. The author discusses the basic definitions, the differing telescope control operating systems, observatory managers, as well as a few current scientific applications.

\section{Introduction}

Early designs of what can be considered precursors to robots started in the First Century A.D. in Byzantium, Alexandria, and other places, but the first automatons called "robots" appeared in 1921 following the name of "robot" by the Czech writer Karel Čapek who took it from his brother Josef [1]. See Figure 1. Years later (1941), Isaac Asimov coined the word "Robotics" [2] for the science and technology of robots, and their design, manufacture, and application.

But what is a robot? Hereby the author introduces some widely accepted definitions.

Robot: A mechanical system that executes repetitive preprogrammed remote tasks with good accuracy with human assistance. Example: Industrial robotic arm.

Teleoperated Robot: A mechanical system that executes various remote tasks with good accuracy and whose behaviour can be dynamically modified with human assistance. Example: Submarine research robots.

Autonomous Robot: A mechanical system which executes various remote simple tasks with good accuracy and is able to adapt itself to changes during the task execution without any kind of human assistance. Example: Rovers devoted to planetary research.
Intelligent Robot: An automatic device which is capable of performing many complex tasks with seemingly human intelligence.

The first attempts to robotize telescopes were developed by astronomers after electromechanical interfaces to computers became common at observatories. Computer control is the most powerful technique for research today, but computer systems are inherently low voltage and are very suspectable to electrical noise. Thus putting electromechanical devices under computer control was particularly challenging. Early examples were expensive, had limited capabilities, and included a large number of unique subsystems, both in hardware and software. This contributed to a lack of progress in the development of robotic telescopes early in their history, but the situation was going to change rapidly.

Similarly, the abovementioned definitions can be extended to Robotic Astronomical Observatories (following the consensus reached after 1 hour of discussion amongst the 80 participants who attended the "Workshop in Robotic Autonomous Observatories," held in Málaga (Spain) on May 18-21, 2009) as follows.

Automated Scheduled Telescope [Robot]: A telescope that performs pre-programmed observations without immediate help of a remote observer (e.g., avoiding an astronomer moving of the mount by hand). 


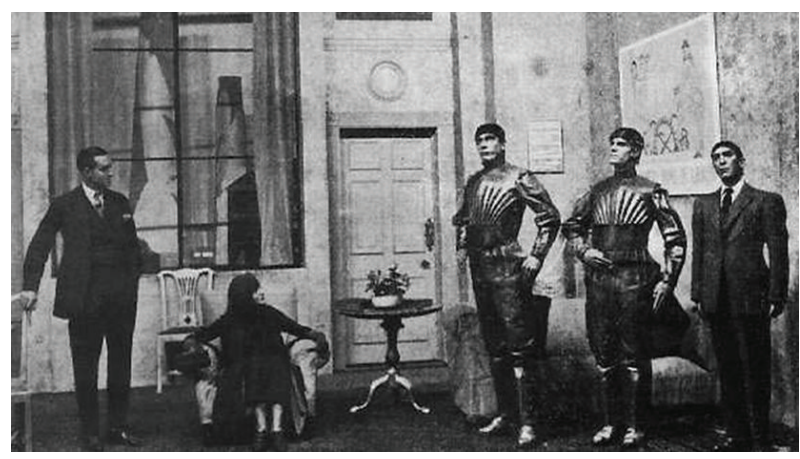

FIgURE 1: A scene in Čapek's play Rossum's Universal Robots (1921) showing three robots, adapted from [4].

Remotely Operated (remote) Telescope [Teleoperated Robot]: A telescope system that performs remote observations following the request of an observer.

Robotic Autonomous Observatory [Autonomous Robot]: A telescope that performs various remote observations and is able to adapt itself to changes during the task execution without any kind of human assistance (e.g., weather monitoring; the system must not endanger a human!).

Robotic Intelligent Observatory [Intelligent Robot]: A robotic observatory in which decisions will be taken by an artificial intelligent system. The next step forward!

\section{Robotic Autonomous Observatories: A Brief History}

In the light of the abovementioned facts and definitions, we can establish the following approximate chronology.

2.1. 1968-1975: The Automated Scheduled Telescopes. The history of robotic telescopes is closely related to the development of automated photoelectric telescopes (APTs). The first one was built by A. C. Code and some of his associates at the University of Wisconsin. A prototype was built by A. Code by the mid 1960s and operated for two or three nights before failing. Once repaired, it operated for other few nights, and was used by B. Millis for gathering data on Cepheids for his Dissertation. In 1968, they used one of the first minicomputers, a DEC PDP- 8 with $4 \mathrm{~K}$ of RAM, for controlling a $0.2 \mathrm{~m}$ reflector telescope with a photometer placed at the prime focus (see Figure 2). The instrument was identical to the one used in the Orbiting Astrophysical Observatory Program: Code envisioned the use of telescopes in the space and later became codirector of the Space Telescope Science Institute. The instrument was used to measure a fixed sequence (from punched paper tape) of bright stars throughout the night to determine the nightly extinction for the larger telescopes [3] and can be considered the first automated scheduled telescope.

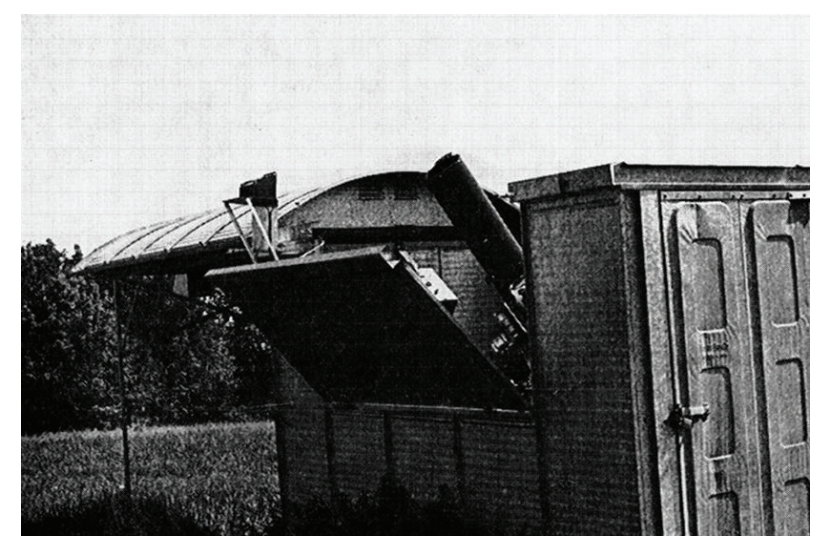

Figure 2: The first computer-controlled telescope ever built, at University of Wisconsin, 1968, adapted from [3].

By the same time (c.1968), the 50-inch B\&C telescope in Kitt Peak was automated ... for one night: the system was too complex and not reliable enough. A $0.15 \mathrm{~m}$ f/8 Cassegrain telescope was the next one to be automated in 1970 [5]. All together, they paved the way and several publications on the field were already available by 1975 [6-8].

2.2. 1975-1984: The Remotely Operated Telescopes. In 1975, a $0.75 \mathrm{~m}$ telescope was fully automated allowing remote operation via a microwave link [9] and in 1976-1977 the Big Telescope Alt-azimuthal $6 \mathrm{~m}$ telescope in Caucasus [10] and the Wyoming InfraRed Observatory $2.3 \mathrm{~m}$ telescope [11] became the first large telescopes to be controlled by a computer. In the late 1970s an IBM 1800 was used by G. Henry and K. Kissell for automated photometry whereas the $0.9 \mathrm{~m}$ Indiana University telescope was computerized by $\mathrm{K}$. Honeycutt et al. See also [12].

Following previous works in late 1970s and early 1980s, the first system which executed repetitive predefined tasks was achieved on the night of 13 of October 1983, when Boyd and Genet run the Phoenix T1 $0.25 \mathrm{~m}$ diameter telescope in Boyd's backyard observatory in Phoenix, Arizona [13]. Boyd and Genet watched the telescope automatically find center and make UBV photometric measurements on star after star and finally went to bed. The telescope ran successfully all night, and almost every clear night for the next quarter century. It was recently retired. Also in 1983, M. Bartels computerized a small telescope using a Commodore 64 computer.

Two years later (1985), a book by Trueblood and Genet was a landmark engineering study in the field [14]. One of this book's achievements was pointing out many reasons, some quite subtle, why telescopes could not be reliably pointed using only basic astronomical calculations. The concepts explored in this book share a common heritage with the telescope mount error modelling software called Tpoint, which emerged from the first generation of large automated telescopes in the 1970s, notably the $3.9 \mathrm{~m}$ Anglo-Australian Telescope (Figure 3).

2.3. 1984-2000: The Era of Autonomous Robotic Observatories. The Carlsberg Meridian Circle $(0.18 \mathrm{~m}$ diameter $)$ in 


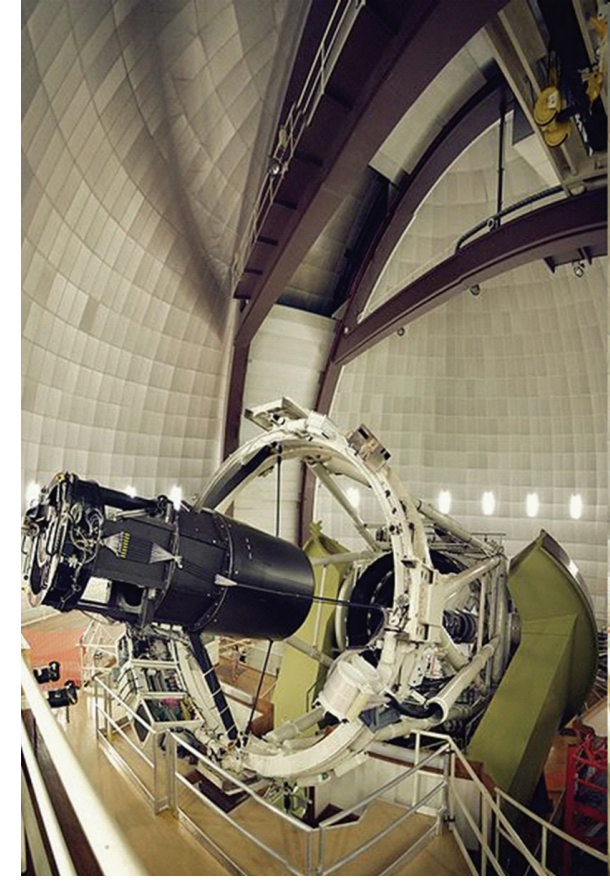

Figure 3: The $3.9 \mathrm{~m}$ Anglo-Australian Telescope is one of the first ones which made use of Tpoint, adapted from [15].

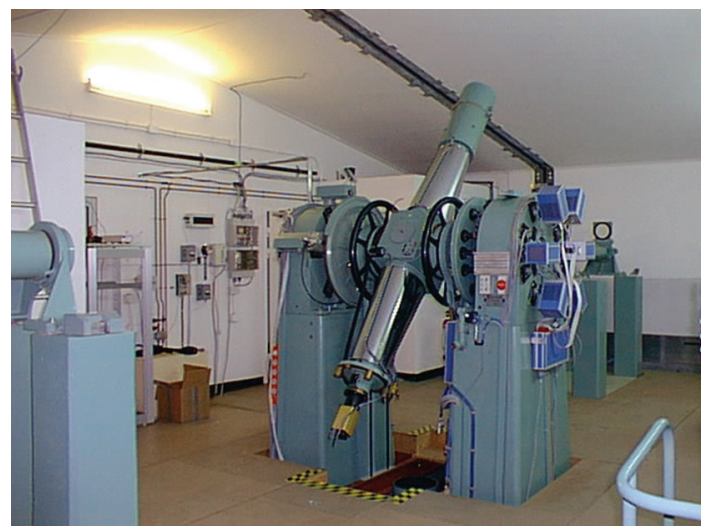

FIgure 4: The Carlsberg Meridian Circle in La Palma was amongst the first automated telescopes, something achieved in 1984. It was until recently operated jointly by the Copenhagen University Observatory, the Institute of Astronomy, Cambridge, and the Real Instituto y Observatorio de la Armada en San Fernando (ROA); however it is now run solely by the ROA. Image was kindly provided by ROA.

La Palma (Canary Islands, Spain) was jointly automated by Copenhagen University Observatory (Denmark) and the Real Instituto y Observatorio de la Armada en San Fernando (Spain) and became in 1984 one of the very first autonomous observatories [16]; see Figure 4.

The first automated telescopes in autonomous observatories were able to start on a preprogrammed sequence of photometric measurements if the sky was clear. This was the case of the Automated Photoelectric Telescope (APT) service, a computer-driven system in Mount Hopkins (U.S.) which

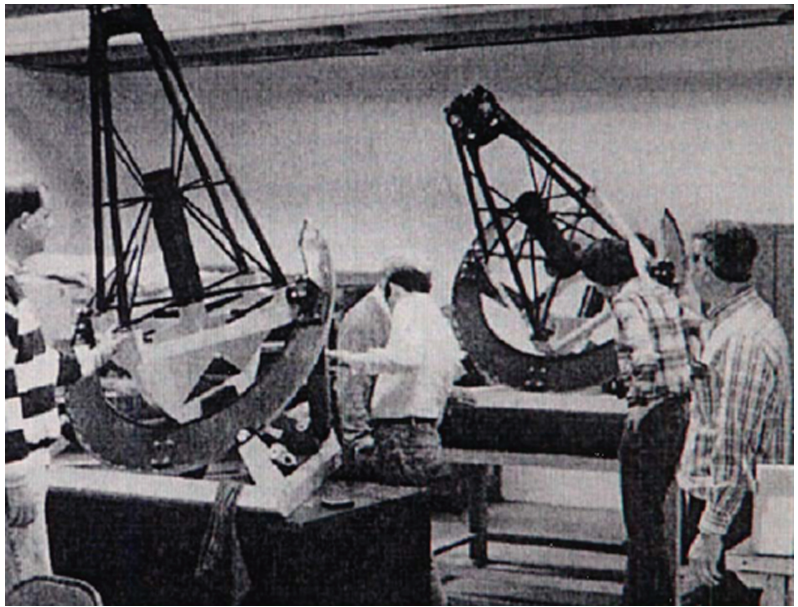

Figure 5: Early APT units.

knew when the Sun set and checked for rain snow, and so forth [17]. See Figure 5.

The Fairborn 10 T2 $0.25 \mathrm{~m}$ APT began operations in early 1984 in Fairborn, Ohio, and was moved in early 1986 to the Fred Lawrence Whipple Observatory (FLWO) on Mount Hopkins in southern Arizona, which was operated jointly by the Harvard Smithsonian Center for Astrophysics and the University of Arizona. It was relocated in 1996 to Fairborn Observatory's new site at $1675 \mathrm{~m}$ in the Patagonia mountains near Washington Camp, Arizona. Operation of the Fairborn $0.25 \mathrm{~m}$ APT [18] was supported by FLWO (during its tenure on Mount Hopkins), Fairborn Observatory, and Tennessee State University. Until 2001 (16 years), the telescope was dedicated primarily to long-term photometric monitoring of semiregular pulsating variable stars and was decommissioned in 2007. Nowadays the TSU group has built 13 instruments including a $2 \mathrm{~m}$ class telescope.

In 1987-88 Genet managed to control a telescope with commands sent over internet. In $1988 \mathrm{M}$. Bartels used an IBM 286 AT to control Alt/Az devices. The same year Gray computerized an equatorial mount and Taki described a twostar Alt/Az alignment routine [19].

Thus, the first robots were telescopes with an absolute positioning control and guiding systems. Observatories with automatic weather stations were gradually being introduced in astronomical observatories.

The Berkeley Automated Imaging Telescopes $(0.5 \mathrm{~m}$ and $0.76 \mathrm{~m}$ diameter telescopes) were used at the astronomy department's Leuschner Observatory in 1992 and 1994 for detailed monitoring of transient objects and for conducting the Leuschner Observatory Supernova Search (LOSS). The $0.76 \mathrm{~m}$ Katzman Automated Imaging Telescope (KAIT) in Lick Observatory (Figure 6) saw first light in 1998 and is still working nowadays and has proven to be very successful in the search for nearby supernovae [20].

The Perugia University Automated Imaging Telescope $(0.4 \mathrm{~m}$ diameter; see Figure 7$)$ in Italy started monitoring blazars in 1992 [21], nearly simultaneously with the $0.8 \mathrm{~m}$ Catania Automatic Photoelectric Telescope [22]. The 


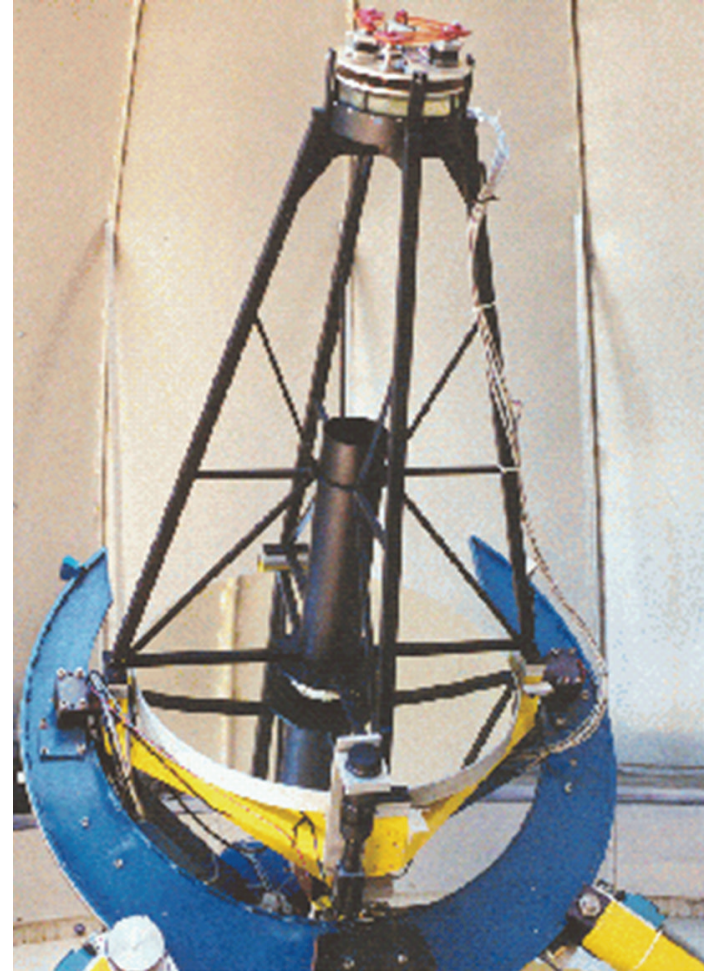

Figure 6: The $0.76 \mathrm{~m}$ KAIT telescope, adapted from [28].

Bradford Robotic Telescope (UK) has been operating via the web since 1993 and accepts requests from anyone and could be considered as one of the first instruments entirely devoted to educational purposes [23]. The $0.5 \mathrm{~m} \mathrm{f} / 1$ Automated Patrol Telescope [24] run by the University of South Wales (Australia) and the University of Iowa Robotic Telescope Facility (0.37 m diameter Rigel telescope at Winer Observatory in AZ [25]) joined later (1994). The second one was devoted to undergraduate education and independent research projects.

In 1997, one of the first robotic amateur observatories achieved first light: the Hanna City Robotic Observatory, which made use of a $0.2 \mathrm{~m}$ Schmidt-Cassegrain telescope [26]. And at about the same time, the CWRU Nassau station $0.9 \mathrm{~m}$ telescope was probably among the first professionalclass instrument publicly available over the internet [27].

And by the end of the 1990s the number of automated telescopes (mostly professional ones) increased substantially with many of them devoted to gamma-ray burst (GRB) followup observations: GROSCE (1993, a wide-field lens system prototype which later gave rise to ROTSE, in the U.S. [29]), LOTIS (1996, a wide-field lens system in Lawrence Livermore Laboratory in the U.S. [30]), ROTSE (1998, a wide-field lens system starting in Los Alamos National Laboratories, U.S. [31]), BOOTES (1998, starting initially with a $0.2 \mathrm{~m}$ telescope and a wide-field lens system located in south Spain [32]), TAROT (1998, a robotic $0.25 \mathrm{~m}$ telescope in France [33], and BART (2000, a $0.2 \mathrm{~m}$ telescope wide-field system in Czech Republic [34]).

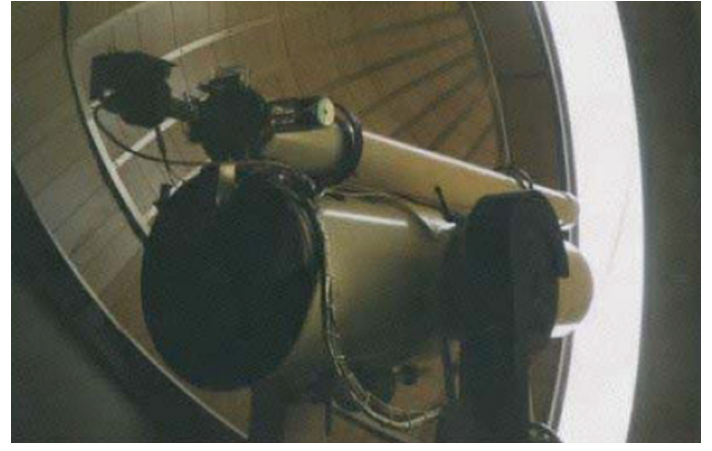

Figure 7: The $0.4 \mathrm{~m}$ Automatic Imaging Telescope of Perugia University, adapted from [42].

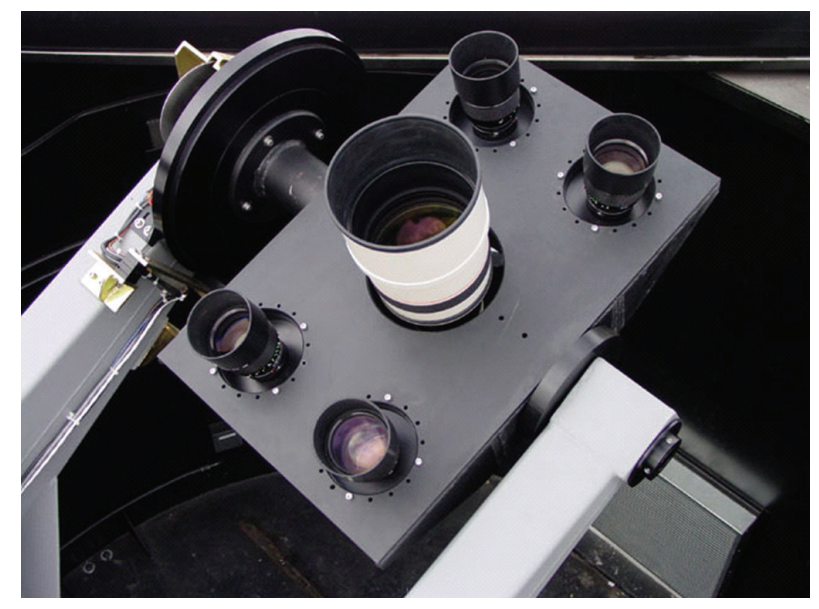

FIGURE 8: The RAPTOR array of wide-field lenses in LANL (U.S.), adapted from [43].

During this decade, several global networks of automated telescopes were proposed by several scientists [35-41]. Although none of them became a reality, their ideas inspired others for the next decade.

\subsection{1-Present: The Era of Robotic Autonomous Observato-} ries Networks. A plethora of robotic autonomous observatories (hereafter RAOs) had first light in the first decade of the 21st century: RAPTOR (2002, an array of wide-field lenses of different diameter [44] in the U.S.; see Figure 8), REM (2003, a $60 \mathrm{~cm}$ robotic nIR telescope in Chile [45]), and many others which we cannot discuss here and are quoted in [46].

They were joined as well by the first global networks: ROTSE-III (four $0.45 \mathrm{~m}$ telescopes in Australia, Namibia, Turkey, and U.S.), BOOTES (four $0.60 \mathrm{~m}$ telescopes in New Zealand, Russia, and two in south Spain, with one devoted to optical/near-IR wavelengths), TAROT (two $0.25 \mathrm{~m}$ telescopes in France and Chile), MASTER (several $0.20-0.35 \mathrm{~m}$ widefield instruments across Russia [47]), ROBONET (three $2.0 \mathrm{~m}$ telescopes in Hawaii, Australia, and Canary Islands [48]), and MONET (two $1.2 \mathrm{~m}$ telescopes in the U.S. and South Africa [49]). 


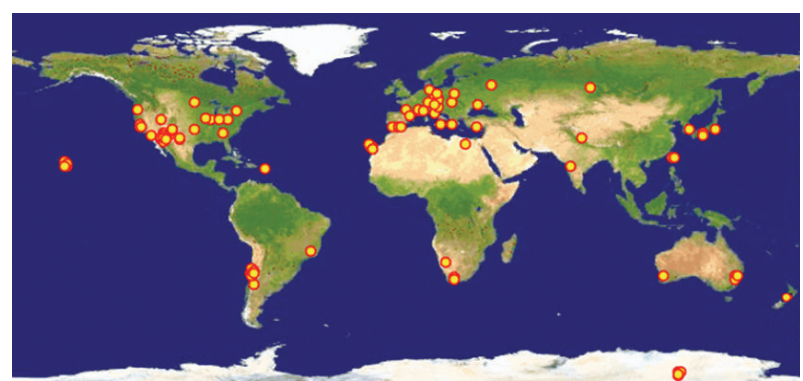

FIgURE 9: The robotic telescopes of the world, adapted from [46].

How are RAOs distributed worldwide? The number of RAOs on Earth is increasing with more that 100 so far of which nearly $80 \%$ are in Europe and in the U.S. (see Figure 9). It is strange why there are no robotic instrumentations in some of the best astronomical locations in the North Hemisphere like Mount Maidanak (Uzbekistan), Khurel Togoot (Mongolia), or San Pedro Mártir (México).

\section{Science and Technology with Robotic Autonomous Observatories}

3.1. Wide Range of Scientific Research Areas. Many scientific fields are benefiting from the use of Robotic Autonomous Observatories. For instance, the Lincoln Near-Earth Asteroid Research (LINEAR) $1 \mathrm{~m}$ telescope was used starting in 1998 for detecting and cataloging near-Earth asteroidsalso referred to as near-Earth objects (NEOs) — that threaten the Earth. By 2009, 226,000 asteroids, 2019 NEOs, and 236 comets had been discovered [50]. Later on, the Moving Object and Transient Event Search System (MOTESS) started as an amateur project in the early 2000s and discovered hundreds of asteroids and a few comets by using the driftscan imaging technique. This project was later used to search for variable stars in $300 \mathrm{sq}$. deg down to $R=19$ [51].

Further Galactic searches for variable objects have been carried out with the ROTSE-III and BOOTES networks of robotic telescopes (see Figure 10), amongst many others. Besides ROTSE-III successful searches for GRB afterglows, BOOTES and ROBONET are both being used for followup observations of GRB and microlensing events.

The Perugian AIT was mainly dedicated to the automatic optical variability monitoring of blazars [52], as well as BART. ROTSE-III has been also used for SN searches and many of the RAOs have also been partially devoted to the followup of transient events like supernovae or GRB afterglows.

An exhaustive list describing the main achievements of RAOs worldwide is beyond the scope of this manuscript. Instead we report the scientific use of RAOs (Table 1) with the number of instruments as a function of the telescope diameter in Table 2.

3.2. Telescope Control Operating Systems. Two kinds are available: Commercial automatization systems (like TCS by Optical Mechanics for apertures in the range 0.4-1 m) which
TABLE 1: Scientific use of RAOs (adapted from [46]).

\begin{tabular}{lc}
\hline Description & Percentage \\
\hline Gamma-ray bursts & $22.1 \%$ \\
Service observations & $15.0 \%$ \\
Education & $14.3 \%$ \\
Photometric monitoring & $10.0 \%$ \\
All-sky surveys & $8.6 \%$ \\
Exoplanet searches & $7.9 \%$ \\
Supernovae search & $7.1 \%$ \\
Asteroids & $5.7 \%$ \\
Spectroscopy & $2.9 \%$ \\
Astrometry & $2.9 \%$ \\
AGN, Quasars & $2.9 \%$ \\
Microlensing & $0.7 \%$ \\
Other uses & $5.7 \%$ \\
\hline
\end{tabular}

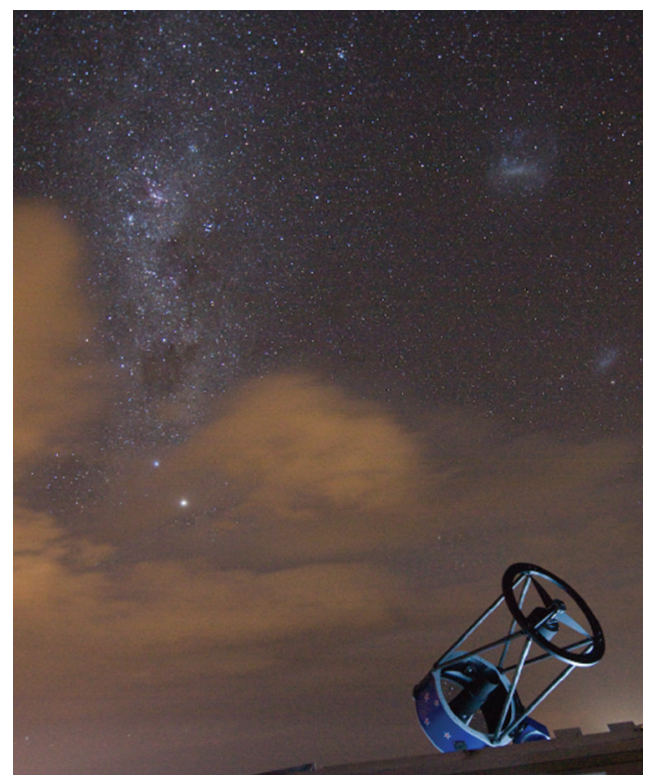

Figure 10: The Yock-Allen $0.6 \mathrm{~m}$ Robotic Telescope at the BOOTES-3 astronomical station in Blenheim (New Zealand) with the Alpha and Beta Centauri, the Southern Cross, and Magellanic Clouds in the background. Photo courtesy of Dietzel.

TABLE 2: Range of RAO's main instrument apertures (adapted from [46]).

\begin{tabular}{lcc}
\hline Description & Number & Percentage \\
\hline$\leq 0.25 \mathrm{~m}$ & 77 & $44.3 \%$ \\
$0.25 \mathrm{~m}<$ aperture $\leq 0.50 \mathrm{~m}$ & 37 & $23.0 \%$ \\
$0.50 \mathrm{~m}<$ aperture $<0.75 \mathrm{~m}$ & 14 & $8.6 \%$ \\
$0.75 \mathrm{~m}<$ aperture $<1.00 \mathrm{~m}$ & 17 & $9.8 \%$ \\
$1.00 \mathrm{~m}<$ aperture $<1.25 \mathrm{~m}$ & 7 & $4.0 \%$ \\
$>1.25 \mathrm{~m}$ & 18 & $10.3 \%$ \\
\hline
\end{tabular}


can be either Open or Closed source and specific control systems (like the one for the $10.4 \mathrm{~m}$ GTC telescope at La Palma, Figure 11).

\subsection{Observatory Managers. There are several observatory} managers already available.

ATIS (Automatic Telescope Instruction Set). It is designed initially in 1984 by Boyd et al. (Arizona) and expanded over the years [53]; ATIS allows many options for real time, lowlevel AI type of control for both aperture photometry, and CCD imaging. The overall system includes provisions for weather sensors, observatory control, and so forth.

OCAAS (Observatory Control and Astronomical Analysis Software). It was originally developed starting in 1993 by Downey and Mutel [54]. In 2001, the software was purchased by Torus Technologies of Iowa City, Iowa. In late 2002, Torus was purchased by Optical Mechanics, Inc., and the updated OCAAS package was renamed as TALON and released under the GPL.

AUDELA. It is developed by Klotz et al. (Toulouse, France), starting in 1995 [55]. Open source code. Linux/Windows.

ASCOM. It is designed in 1998, by B. Denny (USA), as an interface standard for astronomical equipment, based on MS's Component Object Model, which he called the Astronomy Common Object Model [56]. Mostly used by amateur astronomers, has been also used by professionals. Windows. Widely used in SN, MP searches.

RTS2. The Robotic Telescope System version 2. is being developed by Kubánek et al., (Ondřejov/Granada) starting in 2000 [57, 58]. Open source code. Linux/Windows (command line and graphical interface foreseen). Widely used in GRB searches.

INDI. The Instrument Neutral Distributed Interface (INDI) was started in 2003 [59]. In comparison to the Microsoft Windows centric ASCOM standard, INDI is a platform independent protocol developed by E. C. Downey (USA). Open source code. Not so widely spread as the upper layer interface was not done.

Open- or Close-Loop Systems. In an open-loop system, a robotic telescope system points itself and collects its data without inspecting the results of its operations to ensure that it is operating properly. An open-loop telescope is sometimes said to be operating on faith, in that if something goes wrong, there is no way for the control system to detect it and compensate.

A closed-loop system has the capability to evaluate its operations through redundant inputs to detect errors. A common such input would be the capability of evaluating the system's images to ensure that it was pointed at the correct field of view when they were exposed.

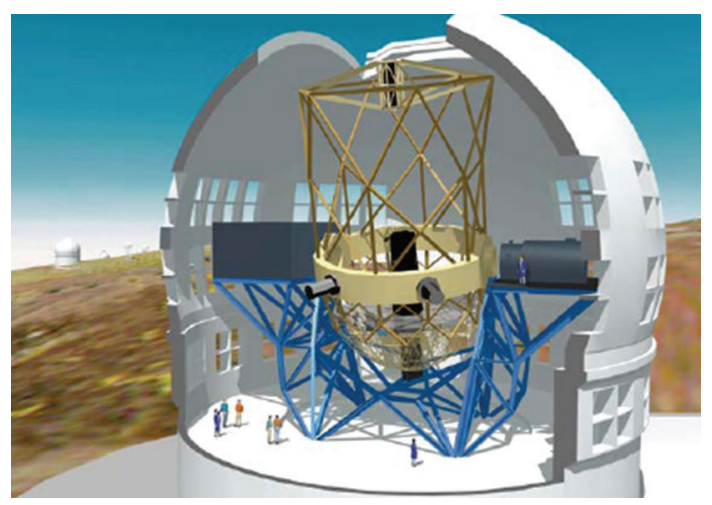

Figure 11: The Spanish 10.4 m GTC telescope in La Palma, Canary Islands. Courtesy of GRANTECAN.

\section{Massive Data Production and Data Analysis}

Until all of the data are analyzed, the robotic observations are worthless. Are all researchers prepared to handle the huge data flow? For instance, the data production of fast imager devices attached to a robotic telescope, could be as high as $\sim 2$ TB/night.

Archiving the data is also most essential. In this era, new generations of powerful tools are being built that rely on reaching transparently across the Internet to query remote services. In order to provide a truly useful service, RAOs should make their data archive available to such tools. The International Virtual Observatory Alliance (IVOA) [60] exists to develop interoperability standards, and RAOs should develop our archive access portal in accordance with those standards [61, 62].

Automatic pipelines able to deliver reliable data products written by capable analysts and computer programmers are a must in order to deliver the data products timely [63, 64]. The real-time analysis of the data is mandatory if we want to fully exploit the data and to be able to alert other observatories for followup observations (see, e.g., [65]).

\section{Education and Public Outreach}

An important mission of any robotic telescope could be the possibility of using a fraction of its time for education and public outreach purposes. In this respect, Robotic telescopes can be operated in two ways:

(i) Direct Mode: remote control with the user directly controlling the telescope using a web/IP cam checking also for weather conditions, and so forth,

(ii) Service Mode: where the user requests and observation is taken by the telescope and returned at a later date. Often called queue scheduling.

In Direct Mode, one person has control of the telescope, although others can watch the system operating. The observer receives the image as soon as the telescope has made the observation. This is most essential in case of real-time demonstrations. 
In Service Mode, each person adds his request to the "input pool". The queue-scheduling computer system then sorts and grades the requests according to the observing details and weather conditions. It aggregates similar requests and creates an observing schedule that it passes to the telescope system. When the observation has been made, an email should be automatically sent to the user who made the request giving details of the location of the image file(s).

\section{The Future Beyond 2010: The Era of Intelligent Robotic Astronomical Networks}

This is the following step, where human assistance in the taking of decisions will be placed by an artificial intelligent system. This is starting to be developed nowadays [66].

Where to get next? With some instruments already planned for Antarctica [67], the far side of the moon is very promising, where straylight and electromagnetic interference are at minimum. Then new drives operating at 1 revolution per month under $1 / 6$ th gravity will need to be designed. Telescope-drive engineers and scientists will go on.

\section{Conclusions}

Since 1984, robotic telescopes have opened a new field in Astrophysics in terms of optimizing the observing time. Most of the existing one-hundred facilities can be considered autonomous robotic observatories (RAOs) with some of them being able to provide prereduced data.

Archiving the data in VO standards and timely analysis (in real-time if possible!) by specific pipelines is not available in most of them, which seriously compromises the new developments of the future intelligent robotic observatories (the next step forward).

In any case, the big advantage of RAOs is that they can be placed in remote locations where human life conditions will be hostile: Antarctica now [68] and the Moon (why not?) in the near future.

\section{Acknowledgments}

The author is very much indebted to R. Genet and P. Abrahams for the very useful suggestions regarding the early times. He also benefited from discussions with R. Cunniffe, J. Gorosabel, S. Guziy, F. Hessman, M. Jelínek, P. Kubánek, and A. de Ugarte Postigo. This work was supported by Spanish Ministry of Science and Technology's projects AYA 200401515, AYA 2007-63677, and AYA 2009-14000-C03-01.

\section{References}

[1] K. Čapek, R.U.R. (Rossum's Universal Robots), Dover, 1921, translated into English in 2001 by P. Selver and N. Playfair.

[2] I. Asimov, "Liar!". Astounding Science Fiction, May 1941 Issue, reprinted by Cambridge University Press, 1977.

[3] J. F. McNall, J. L. Miedaner, and A. D. Code, "A computercontrolled photometric telescope," Astronomical Journal, vol. 73, pp. 756-761, 1968.
[4] http://www.umich.edu/ engb415/literature/pontee/RUR/ RURsmry.html.

[5] R. H. Lee, R. M. MacQueen, and W. G. Mankin, "A computercontrolled infrared eclipse telescope," Applied Optics, vol. 9, no. 12, pp. 2653-2657, 1970.

[6] E. W. Dennison, "Computer control of large telescopes," in Proceedings of Conference on Large Telescope Design, R. M. West, Ed., ESO/CERN Conference, p. 363ff, Geneva, Switzerland, 1971.

[7] E. W. Dennison, "Recent computer installations at the hale observatories," in Automation in Optical Astrophysics, $\mathrm{H}$. Seddon and M. J. Smyth, Eds., Proceedings of IAU Colloq. 11, p. 8ff, Publ. R. Obs., Edinburgh, UK, 1971.

[8] L. B. Robinson, "On-line computers for telescope control and data handling," Annual Review of Astronomy and Astrophysics, vol. 13, pp. 165-185, 1975.

[9] S. A. Colgate, E. P. Moore, and R. Carlson, "A fully automated digitally controlled 30-inch telescope," Publications of the Astronomical Society of the Pacific, vol. 87, pp. 565-575, 1975.

[10] http://w0.sao.ru/Doc-en/Telescopes/bta/descrip.html.

[11] R. D. Gehrz and J. A. Hackwell, "Exploring the infrared universe from Wyoming," Sky \& Telescope, vol. 55, pp. 466473, 1978.

[12] D. Skillman, "Running a telescope with a microcomputer," $S k y$ \& Telescope, vol. 61, p. 71, 1981.

[13] R. M. Genet, "Introduction to automatic photoelectric telescopes (APTs)," International Amateur-Professional Photoelectric Photometry Communication, no. 25, pp. 1-25, 1986.

[14] M. Trueblood and R. Genet, Microcomputer Control of Telescopes, Willmann-Bell, Richmond, Va, USA, 1985.

[15] http://upload.wikimedia.org/wikipedia/commons/thumb/8/ 8c/Aat.jpg/250px-Aat.jpg.

[16] L. Helmer and L.V. Morrison, "Carlsberg automatic meridian circle," Vistas in Astronomy, vol. 28, pp. 505-518, 1985.

[17] S. Baliunas, L. J. Boyd, R. M. Genet, D. S. Hall, and S. Criswell, "Automatic photoelectric telescope III the Mount Hopkins site," International Amateur-Professional Photoelectric Photometry Communication, no. 22, p. 47, 1985.

[18] R. Genet, L. J. Boyd, K. E. Kissell, D. L. Crawford, and D. S. Hall, "The automatic photoelectric telescope service," Publications of the Astronomical Society of the Pacific, vol. 99, pp. 660-667, 1987.

[19] T. Taki, "A new concept in computers-aided telescopes," Sky \& Telescope, vol. 77, p. 194, 1989.

[20] A. V. Filippenko, W. D. Li, R. R. Treffers, and M. Modjaz, "The lick observatory supernova search with the Katzman automatic imaging telescope," in Small-Telescope Astronomy on Global Scales, vol. 246 of ASP Conference Series, pp. 121130, Astronomical Society of the Pacific, San Francisco, Calif, USA, 2001.

[21] G. Tosti, S. Pascolini, and M. Fiorucci, "The Perugia University automatic observatory," Publications of the Astronomical Society of the Pacific, vol. 108, no. 726, pp. 706-713, 1996.

[22] M. Rodonó, G. Cutispoto, A. F. Lanza, and S. Messina, "The Catania automatic photoelectric telescope on Mt. Etna: a systematic study of magnetically active stars," Astronomische Nachrichten, vol. 322, no. 5-6, pp. 333-342, 2001.

[23] J. Baruch, "Bradford robotic telescope: education \& public outreach: journey into space," Astronomy \& Geophysics, vol. 48, no. 4, pp. 4.27-4.28, 2007.

[24] M. C. B. Ashley, http://www.phys.unsw.edu.au/ mcba/apt/.

[25] http://phobos.physics.uiowa.edu/.

[26] http://www.mtco.com/ jgunn/.

[27] http://astroweb.case.edu/history.shtml. 
[28] http://astro.berkeley.edu/ bait/kait.html.

[29] C. W. Akerlof, M. Fatuzzo, B. Lee, et al., "Gamma-ray optical counterpart search experiment (GROCSE)," in Proceedings of the 2nd Workshop on Gamma-Ray Bursts, vol. 307 of AIP Conference Proceedings, pp. 663-666, Huntsville, Ala, USA, 1994.

[30] H. S. Park, E. Ables, S. Barthelmy, et al., "SuperLOTIS/LOTIS/LITE: prompt GRB followup experiments," in GAMMA 2001; Gamma-Ray Astrophysics 2001, vol. 587 of AIP Conference Proceedings, pp. 181-184, 2001.

[31] C. W. Akerlof, R. L. Kehoe, T. A. McKay, et al., "The ROTSEIII robotic telescope system," Publications of the Astronomical Society of the Pacific, vol. 115, no. 803, pp. 132-140, 2003.

[32] A. J. Castro-Tirado, J. Soldán, M. Bernas, et al., "The burst observer and optical transient exploring system (BOOTES)," Astronomy and Astrophysics Supplement Series, vol. 138, no. 3, pp. 583-585, 1999.

[33] M. Böer, M. Bringer, A. Klotz, et al., "TAROT: observing gamma-ray bursts "in progress"', Astronomy \& Astrophysics, vol. 138, pp. 579-580, 1999.

[34] M. Jelínek, P. Kubánek, R. Hudec, M. Nekola, M. Topinka, and J. Strobl, "BART-burst alert robotic telescope," in The Astrophysics of Cataclysmic Variables and Related Objects, vol. 330 of ASP Conference Series, p. 481, 2005.

[35] F. R. Querci and M. Querci, “The Network of Oriental Robotic Telescopes (NORT)," African Skies 2, 18, 1998.

[36] E. Budding, "Globalizing observations: prospects and practicalities," in Stellar Photometry-Current Techniques and Future Developments, C. J. Butler and I. Elliott, Eds., IAU Colloquium 136, p. 257, Cambridge University Press, 1993.

[37] D. L. Crawford, "GNAT: global network of automated telescopes," in Automated Telescopes for Photometry and Imaging, vol. 28 of ASP Conference Series, p. 111, 1992.

[38] D. L. Crawford, "Some GNAT issues," in Robotic Observatories, M. F. Bode, Ed., p. 77, John Wiley \& Sons and Praxis, New York, NY, USA, 1995.

[39] F. R. Querci, M. Querci, S. Kadiri, and Z. Benkhaldoun, "Network of Oriental Robotic Telescopes," in Symposium: Robotic Telescopes: Current Capabilities, Present Developments, and Future Prospects for Automated Astronomy, G. W. Henry and M. Drummond, Eds., vol. 79 of ASP Conference Series, Flagstaff, Ariz, USA, June 1995.

[40] F. R. Querci, M. Querci, and S. Kadiri, "A complementary Network to GNAT: an Arabian and French Project for automated photometric stations," in Robotic Observatories, Held in Kilkenny (Ireland), July, 1992, B. P. Hines and M. F. Bode, Eds., p. 85, Praxis and John Wiley \& Sons, New York, NY, USA, 1995.

[41] F. R. Querci and M. Querci, "Robotic telescopes and networks: new tools for education and science," Astrophysics and Space Science, vol. 273, no. 1-4, pp. 257-272, 2000.

[42] http://astro.fisica.unipg.it/osserv.htm.

[43] http://www.raptor.lanl.gov/.

[44] W. T. Vestrand, K. N. Borozdin, S. P. Brumby, et al., "The RAPTOR experiment: a system for monitoring the optical sky in real time," in Advanced Global Communications Technologies for Astronomy II, vol. 4845 of Proceedings of the SPIE, pp. 126136, 2002.

[45] F. M. Zerbi, G. Chincarini, G. Ghisellini, et al., "REM telescope, a robotic facility to monitor the prompt afterglow of gamma ray bursts," in Instrument Design and Performance for Optical/Infrared Ground-Based Telescopes, vol. 4841 of Proceedings of the SPIE, pp. 737-748, 2002.
[46] F. Hessman, "Robotic telescope projects," http://www.astro .physik.uni-goettingen.de/ hessman/MONET/links.html.

[47] V. M. Lipunov, V. G. Kornilov, A. V. Krylov, et al., "The master mobile astronomical system. Optical observations of gammaray bursts," Astrophysics, vol. 48, no. 3, pp. 389-399, 2005.

[48] http://www.astro.ljmu.ac.uk/RoboNet/.

[49] http://monet.uni-goettingen.de/.

[50] http://www.ll.mit.edu/mission/space/linear/.

[51] A. L. Kraus, E. R. Craine, M. S. Giampapa, W. W. G. Scharlach, and R. A. Tucker, "The first MOTESS-GNAT variable-star survey," Astronomical Journal, vol. 134, no. 4, pp. 1488-1502, 2007.

[52] http://astro.fisica.unipg.it/PGblazar/tabella2000.htm.

[53] R. M. Gener, L. J. Boyd, and D. S. Hayes, "Automatic Small Telescope Operations," in New Directions in Spectrophotometry, A. G. Davis Philip, D. S. Hayes, and S. J. Adelman, Eds., L. Davis Press, Schenectady, NY, USA, Las Vegas, Nev, USA, March 1988.

[54] E. C. Downey and R. L. Mutel, "The university of Iowa automated telescope facility," in Astronomical Data Analysis Software and Systems V, G. H. Jacoby and J. Barnes, Eds., vol. 101 of ASP Conference Series, p. 380, 1996.

[55] http://www.audela.org/english_audela.php.

[56] http://ascom-standards.org/.

[57] P. Kubánek, M. Jelínek, J. French, et al., "The RTS2 protocol," in Advanced Software and Control for Astronomy II, A. Bridger and N. M. Radziwill, Eds., vol. 7019 of Proceedings of the SPIE, pp. 70192S-1-70192S-12, 2008.

[58] http://rts2.org/.

[59] http://www.indilib.org/.

[60] http://www.ivoa.net/.

[61] http://www.ivoa.net/Documents/SIA/.

[62] http://www.ivoa.net/Documents/latest/CharacterisationDM .html.

[63] P. Ballester, P. Biereichel, A. Kaufer, M. Kiekebusch, and H. Lorch, "CLIP: bridging pipelines to instrument control software," in Astronomical Data Analysis Software and Systems, vol. 394 of ASP Conference Series, pp. 577-580, 2008.

[64] C. Rité, R. Slijkhuis, P. Rosati, et al., "Production of previews and advanced data products for the ESO science archive," in Astronomical Data Analysis Software and Systems, vol. 394 of ASP Conference Series, pp. 605-608, 2008.

[65] D. L. Starr, J. S. Bloom, and N. R. Butler, "Real-time transient classification and broadcast pipeline," in Astronomical Data Analysis Software and Systems, vol. 394 of ASP Conference Series, pp. 609-612, 2008.

[66] R. R. White, S. M. Evans, W. T. Vestrand, M. S. Warren, J. A. Wren, and P. R. Wozniak, "Thinking telescopes and the future astronomical meta-network," Astronomische Nachrichten, vol. 327, no. 8, pp. 758-762, 2006.

[67] M. Busso, G. Tosti, F. Roncella, et al., "The IRAIT project infrared astronomy from Antarctica," EAS Publications Series, vol. 14, pp. 181-186, 2005.

[68] M. C. B. Ashley, M. G. Burton, J. S. Lawrence, and J. W. V. Storey, "Robotic telescopes on the Antarctic plateau," Astronomische Nachrichten, vol. 325, no. 6, pp. 619-625, 2004. 

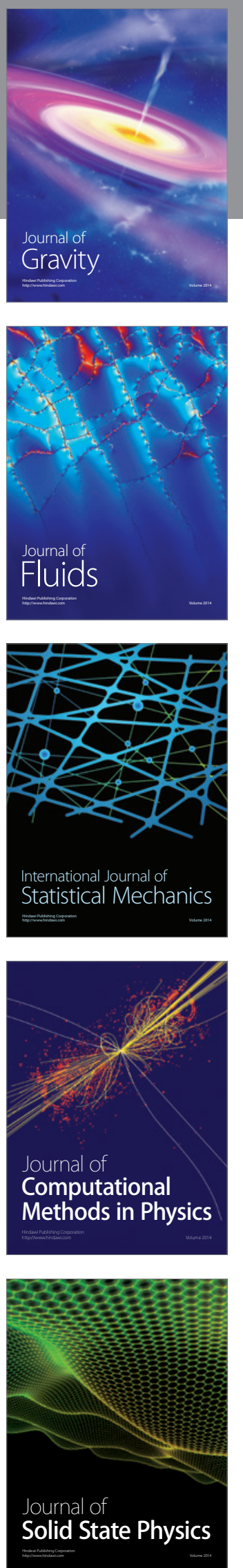

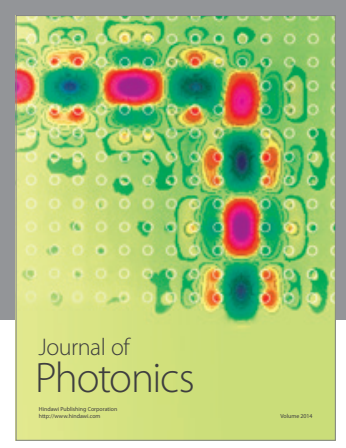

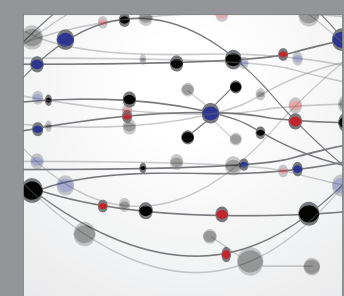

The Scientific World Journal
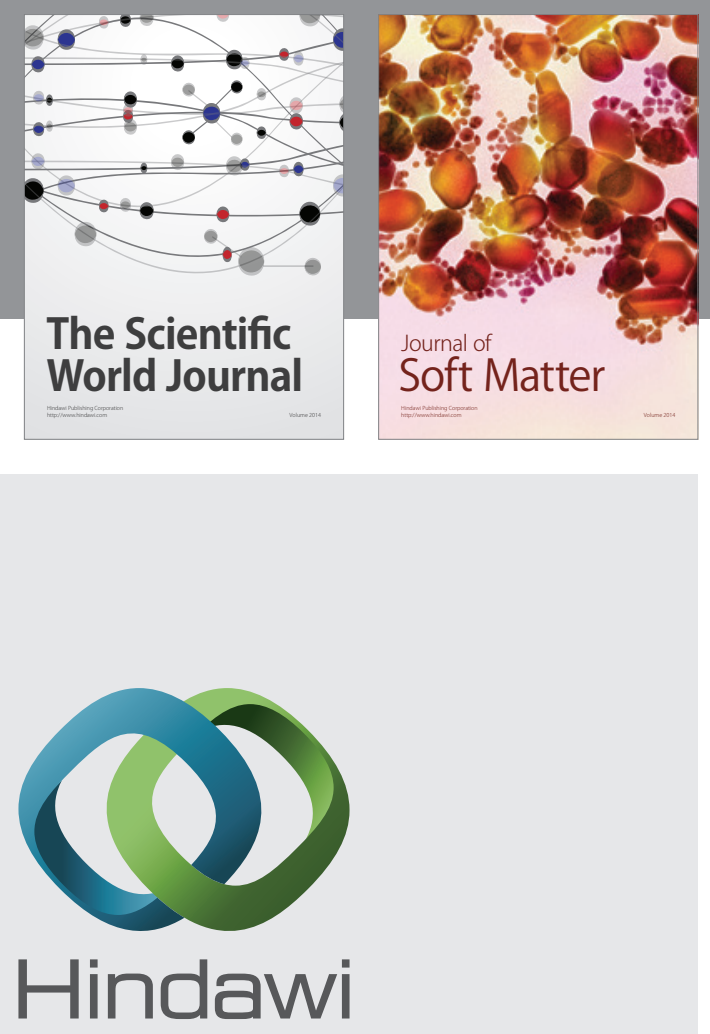

Submit your manuscripts at

http://www.hindawi.com
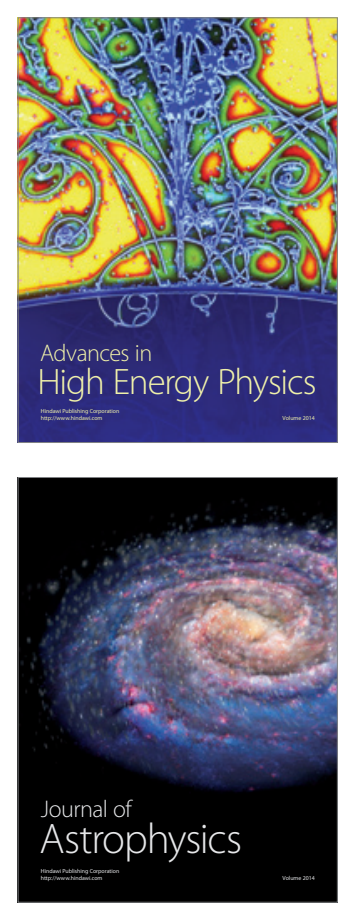
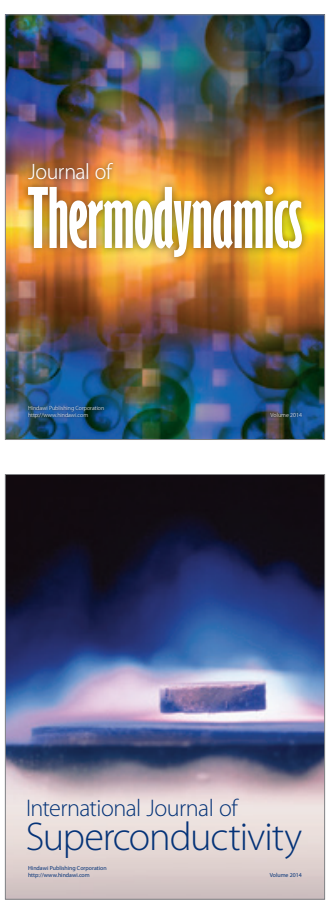
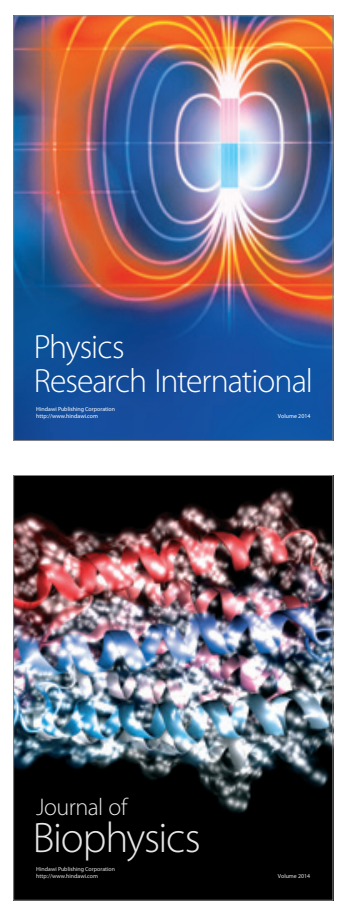
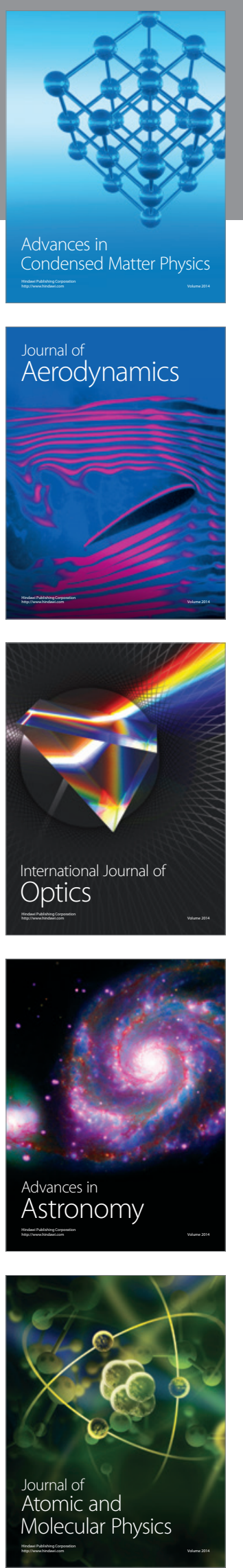